\title{
An Overview on the Sequential Treatment of Pancreatic Neuroendocrine Tumors (pNETs)
}

Teresa Alonso-Gordoa · Juan José Díez · Javier Molina •

Pablo Reguera · Olga Martínez-Sáez • Enrique Grande

To view enhanced content go to www.rarecancers-open.com

Received: May 19, 2015 / Published online: July 11, 2015

(C) The Author(s) 2015. This article is published with open access at Springerlink.com

\section{ABSTRACT}

Patients suffering from pancreatic neuroendocrine tumors (pNETs) are now candidates to receive novel approved drugs that have demonstrated benefit in disease control rate and delay the time taken for tumor progression in Phase III clinical trials; for example, sunitinib, everolimus and lanreotide. Though pNETs represent a rare and heterogeneous disease, recent approaches are being taken to better understand the molecular pathways involved in carcinogenesis. Consequently, new treatment strategies are now available and others still under investigation show promising results. However, some questions around how to approach patients with pNETs are still unresolved, such as what the

Electronic supplementary material The online version of this article (doi:10.1007/s40487-015-0007-6) contains supplementary material, which is available to authorized users.

T. Alonso-Gordoa $(\varangle) \cdot J$. Molina · P. Reguera ·

O. Martínez-Sáez · E. Grande

Medical Oncology Department, Ramón y Cajal

University Hospital, Ctra. Colmenar Viejo km 9100,

28034 Madrid, Spain

e-mail: talonso@oncologiahrc.com

J. J. Díez

Endocrinology and Nutrition Department, Ramón y

Cajal University Hospital, Madrid, Spain best sequence of treatments we can offer to each of our patients in the clinic at any time of their disease would be. Therapeutic decisions are, at the moment, guided by clinical judgment, based on different parameters coming from retrospective analysis and non-randomized clinical trials. However, advances in genomic research would lead to a more precise approach using therapeutic targets that would also allow the development of new agents, prognostic or predictive biomarkers and a better understanding of resistance mechanisms. The following article is a comprehensive review of the approved and investigational drugs in pNET, and highlights the current concerns about treatment sequencing, but also provides an update of some of the present and future efforts for an improvement in the therapeutic algorithm of the disease.

Keywords: Chemotherapy; Molecular biology; Pancreatic neuroendocrine tumor; pNET; Resistance mechanisms; Sequencing; Somatostatin analogs; Targeted agents

\section{INTRODUCTION}

A wide spectrum of disease is observed in pancreatic neuroendocrine tumors (pNETs); 
pNETs make up 3\% of primary pancreatic malignancies and $12 \%$ of all gastroenteropancreatic neuroendocrine tumors (GEP NETs) [1]. Five-year overall survival (OS) rates range between $7 \%$ and $75 \%$ for patients with high and low-grade tumors, respectively [2]. Goals of therapy for pNETs include not only the prolongation of life, but also the improvement of disease-related symptoms, and control of burden of disease and quality of life.

Based on current molecular understanding, several therapies have demonstrated efficacy in pNETs, and novel drugs are still currently being investigated [3]. Treatment sequencing in these tumors is a novel concept, and has arisen from the availability of new, different and effective therapeutic agents. However, the correct place of those therapies within the treatment algorithm at each time of disease progression to achieve maximum impact in OS is still a matter of debate. Clinicians guide their decisions based on different parameters, but accurate biomarkers and information about sequencing coming from clinical trials are still lacking, and the wide spectrum of clinical behaviors in pNETs brings challenges in selecting the best treatment and monitoring response. Therefore, a multidisciplinary approach from expert physicians is essential in this particular group of patients belonging to a rare and complex disease [4].

\section{AN OVERVIEW OF PNETS}

pNETs represent both a heterogeneous and a rare group of tumors with low frequency $(0.32$ diagnosed cases per 100,000 population per year) [5]. However, this figure has been increasing over recent years, as reported in a Japanese survey which showed an estimated prevalence of 2.69 per 100,000 in 2010, which corresponded to an increase of 1.2 times the number of patients with pNETs receiving oncological treatment compared to 2005 [6]. Improvement in diagnostic tools, increase in interest among physicians for the performance of particular radiological and nuclear images, the acquisition of adequate tumor samples, and detailed pathological information have lead to very accurate classification of NETs and, consequently, to a precise therapeutic strategy.

The diagnosis of pNETs is based on clinical symptoms, specific hormonal markers, radiological or endoscopic images, and pathological information. Different classifications for pNETs according to different issues have been proposed. About $90 \%$ of pNETs are functional and some are able to release peptides that are responsible for different hormonal syndromes [4]. Histological findings have been proposed to correlate with prognosis [7]. Thus, the last updated classification from the World Health Organization (WHO) divided NETs into two different categories: (1) welldifferentiated (WD)-NETs G1 (mitotic count $<2 \times 10$ high power field [HPF] and Ki67 index $\leq 2 \%$ ) and G2 (mitotic count 2-20 × $10 \mathrm{HPF}$ and Ki67 index 3-20); and (2) poorly differentiated (PD)-neuroendocrine carcinomas (NECs) G3 (mitotic count $>20 \times 10 \mathrm{HPF}$ and Ki67 >20) [8] Recent advances in nuclear medicine using radiolabelled somatostatin analog (SSA) agents overcome challenging issues in the diagnostic and therapeutic assessment of NETs, such as the development of somatostatin receptor scintigraphy (SRS) using ${ }^{111}$ In-octreotide [9]. Indeed, novel radiopharmaceuticals for use in positron emission tomography (PET) imaging with better resolution have shown to improve the limitations of the previous methods in diagnosis (e.g., location of primary tumor or recurrent disease and identification of cellular 
populations), staging, treatment approach (in loco-regionally confined or intermediate grade tumors), treatment monitoring and prediction of response [10]. A precise histological report is essential in NETs and, in G2-NETs and high grade NECs, the question of whether all patients in each group should be treated the same way is now under debate. Recent findings highlight the heterogeneity within the group of patients harboring high-grade NECs as well as the need for predictive and prognostic factors to select the optimal treatment [11]. Should we consider different treatment schedules according to differentiation and Ki67? Some controversial aspects include the consideration of different subtypes, such as a group of differentiated, but highly proliferative tumors (Ki67 20-50\%; NET Grade 3) and a group of true PD-NECs (Ki67 > 50-55\%; NEC) [12]. These novel groups are not well stratified or represented in earlier clinical trials, and thus definitive conclusions are more difficult to suggest according to a specific group of patients. Current and ongoing clinical trials are trying to solve this problem by focusing the studies in more homogeneous subgroups of patients and stratifying them properly.

Prognosis in pNETs is related to the clinical stage at diagnosis [1]. The European Neuroendocrine Tumor Society (ENETS) TNM staging system describes a 5-year OS rate for stage I, II, III and IV of $100 \%, 88 \%, 85 \%$ and $57 \%$, respectively [1]. The American Joint Committee on Cancer (AJCC) classification establishes a 5 -year OS rate for stage I, II, III and IV of $92 \%$, $84 \%, 81 \%$ and $57 \%$, respectively [1].

\section{CURRENT TREATMENTS FOR PNETS}

Despite the recent availability of a number of drugs for the treatment of pNETs and the extensive field of current research, there are some unresolved issues in the management of tumors that are expected to be elucidated in the near future.

Treatment algorithm for the best treatment approach is still under debate; patients with NETs should be treated with all the available regimens for their precise classification to achieve the maximum prolongation in survival and maintenance of quality of life, together with adequate symptom control. Treatment decisions are based on tumor characteristics (e.g., differentiation, Ki67, mitotic rate), stage, clinical course, tumorrelated symptoms, tumor secretory symptoms, rate of progression, number of affected organs, disease burden, functioning, findings from somatostatin receptors (SSTRs), European Cooperative Oncology Group (ECOG) performance status, comorbidities, and concomitant medication. In fact, there is a lack of data comparing the activity of different treatment strategies, and final decisions are based on personal experience and expert consensus statements [2]. In this context, different questions are still undefined, such as the best treatment sequence and whether all patients may benefit from all available drugs; the reliable prognostic and predictive biomarkers to guide our treatment decisions; whether combination strategies improve efficacy over sequential monotherapy regimens in a safe manner or by increasing efficacy by adding cytotoxic agents to targeted agents to improve the limited response rates of available drugs; the duration of treatment agents; or the real OS benefit of currently used drugs.

Several mechanisms of resistance have been suggested relating to SSAs, such as desensitization or downregulation of SSTRs on the cell surface of tumor cells or its internalization after a prolonged exposure to 
an agonist; different SSTR subtype expression patterns; the development of functioning mutation forms of SSTR; the generation of antibodies against SSA; or changes in regulatory proteins (amphyphysin IIb) that are involved in SSTR stabilization [13]. Different strategies proposed to overcome resistance to SSA include administration of SSAs in a highdose or short-schedule manner; the sequential treatment of both available SSAs (octreotide/ lanreotide); and the development of chimeric SST/dopamine receptor [DR] molecules [14] or targeted agents to multiple SSTR, such as pasireotide [15]. However, none of those strategies have enough strength to allow definitive conclusions. The Cooperate-2 Phase II trial presented at ENETS 2015 did not show a benefit in the primary endpoint in the group of patients treated with everolimus and pasireotide compared with single-agent everolimus (median progression-free survival [PFS] 16.6 versus 16.8 months, hazard ratio [HR] 0.99, 95\%; CI 0.64-1.54; $p=0.488$ ) [16].

The beneficial role of antiangiogenic agents in pNETs is undeniable. However, resistance mechanisms have also been identified in patients treated with targeted therapies based on the activation of alternative pathways [17] by the presence of other angiogenic factors, including vascular endothelial growth factor (VEGF)-independent vessel growth, inflammatory cells releasing cytokines with proangiogenic effect and protecting cells from hypoxia, pericyte coverage, and enhancing invasiveness [18]. Different ways have been proposed to overcome those resistance mechanisms: optimizing the dose of the drug, switching to an alternative VEGF therapy, switching to a drug with a different mechanism of action, or using a combination strategy [19]. Mammalian target of rapamycin (mTOR) inhibitors' evasive resistance involves the activation of alternative pathways or feedback loops (RAS/RAF/Erk pathway), dysregulation of downstream pathways (loss of 4E-BP1 inhibition, eIF4E activation, loss of function of PP2A, p27 levels, cyclin D1 expression) and promotion of autophagy [18]. Potential mechanisms to overcome resistance to mTOR inhibitors are: dual blockade of mTOR complexes 1 and 2 (mTORC1 and mTORC2), combination therapies with an mTOR inhibitor and a second agent that blocks upstream kinases (protein kinase B [AKT] or phosphatidylinositol 3-kinase [PI3 K]) or receptors (insulin growth factor receptor [IGFR]), mTOR/epidermal growth factor receptor [EGFR] dual inhibition [rapamycin/erlotinib] [20], or switch to an agent with a different mechanism of action [21].

Initial trials in NETs showed a trend to a better outcome for patients with pNETs treated with cytotoxic drugs over patients with carcinoid tumors [22]. Alkylating agents were investigated early in the discovery of NETs, and streptozocin (STZ) was the first drug approved by the Food and Drug Administration (FDA) in 1976. Combination therapy with doxorubicin and fluorouracil or single-agent dacarbazine obtained promising overall response rate (ORR) results of up to $70 \%$ in several clinical trials $[23,24]$ (Table 1). However, the outcome criteria used in earlier trials, based on nonradiographic response criteria, are not consistent and comparable with those we rely on today. A more recent trial assessing tumor response with modern criteria showed an ORR of 39\% with STZ-based chemotherapy [25] and an international survey described that this treatment strategy is broadly administered in patients with NETs [26].

Moreover, three targeted agents (Table 1) have been recently approved for the treatment of patients with advanced and progressive pNETs. Interest in antiangiogenic agents 


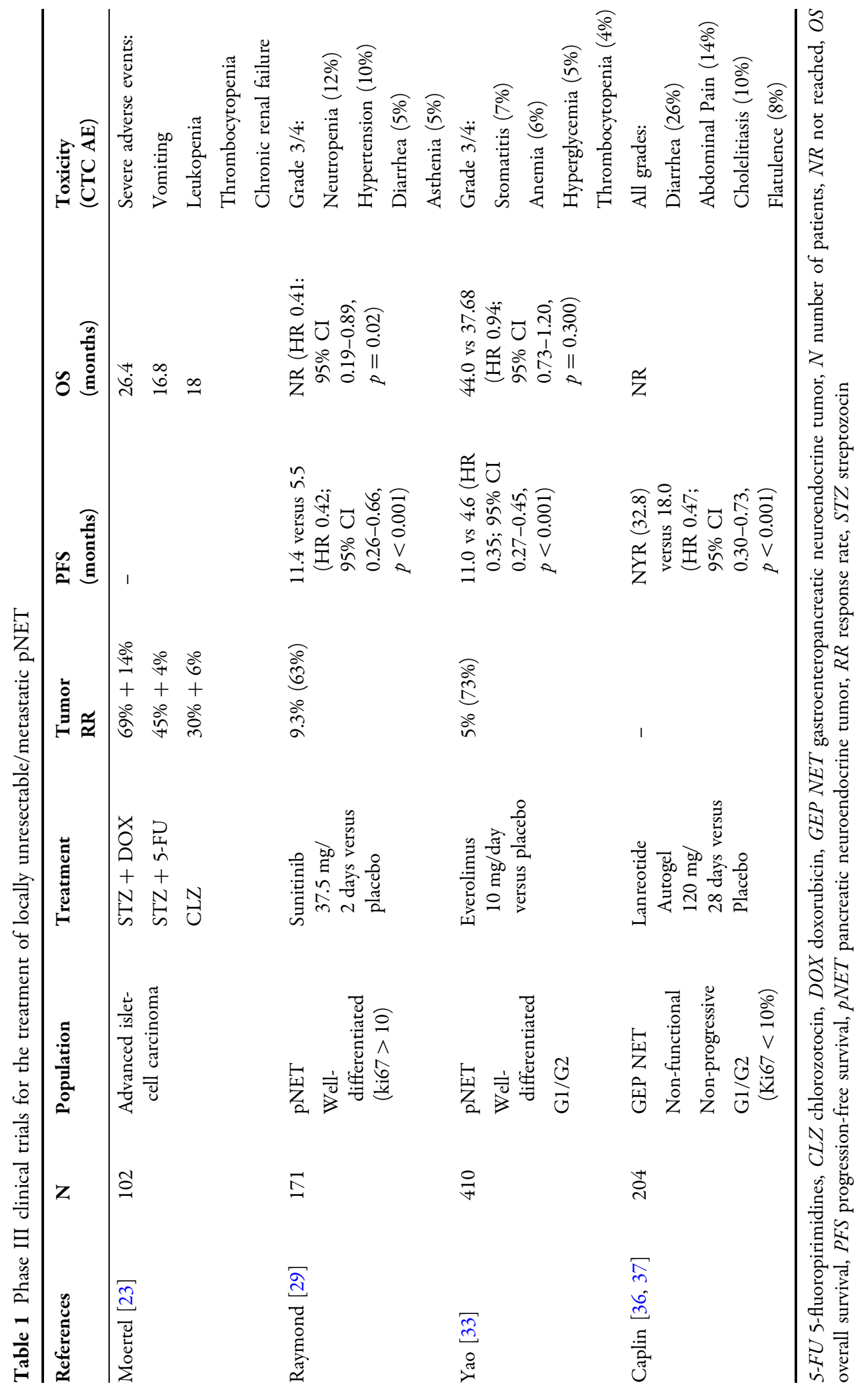


started with the discovery of NETs with high vasculature, and good results in efficacy have been obtained from a Phase I dose escalation study with sunitinib for advanced malignancies in this subgroup of patients [27]. The Phase II trial included 66 patients with pNETs, and showed an ORR of $16.7 \%$ and median time to progression (TTP) of 7.7 months [28]. Finally, the Phase III placebo-controlled trial was conducted, but terminated early due to safety measures relating to an increase in serious adverse events, disease progression and deaths observed in the placebo group [29]. A total of 171 patients were finally randomized to sunitinib versus placebo (1:1) and the benefit in PFS was almost 6 months for the patients in the experimental group (median PFS 11.4 months versus 5.4 months; HR $0.42 ; 95 \%$ CI $0.26-0.66, p<0.001)$.

The mTOR pathway that is involved in the regulatory functions within the tumor and tumor microenvironment also plays an important role in the development of NETs due to gene mutations, such as phosphatase and tensin homolog (PTEN), tuberous sclerosis 2 (TSC2) and PI3 K, catalytic, alpha polypeptide (PI3KCA), or activation of insulin growth factor 1 (IGF1) [30]. The approval of everolimus for the treatment of pNETs following the results of its pivotal Phase III trial was based on the benefit observed in two Phase II previous studies. The first study included 60 patients with naïve or previously treated WD-NETs $(N=30$ patients with pNET) who received treatment with everolimus $5 \mathrm{mg}$ or $10 \mathrm{mg}$ daily and octreotide long-acting repeatable (octreotide LAR) $30 \mathrm{mg}$ monthly [31]. The ORR in pNETs was $27 \%$, and 6- and 12-month PFSs were $73 \%$ and $48 \%$, respectively. The second study was the RADIANT-1 that was conducted to assess the benefit of everolimus $10 \mathrm{mg}$ once daily with or without octreotide LAR in 160 pretreated patients with progressive WD-pNETs [32]. The data were consistent with previous trials: median PFS and OS were 8.5 and 24.9 months for everolimus, respectively. For patients treated with everolimus plus ocreotide LAR, PFS was 15.2 months and OS was not reached. These data showed a trend towards a better outcome with both treatments together. The Phase III RADIANT 3 clinical trial assessed the benefit of everolimus as first line therapy in pNETs; 410 patients were randomized to receive everolimus $10 \mathrm{mg}$ or placebo every $24 \mathrm{~h}$ [33]. The first analysis showed a benefit in the primary endpoint with a median PFS of 11.0 months in the everolimus group and 4.6 months in the placebo group (HR 0.35; 95\% CI 0.27-0.45, $p<0.001)$. The updated outcome results that included the data from the open-label extension phase were presented at the European Society of Medical Oncology (ESMO) 2014 Congress; everolimus obtained 6.3-month longer median OS compared with placebo (44.0 months versus 37.68 months; HR 0.94; 95\% CI 0.73-1.20, $p=0.3$ ) [34].

The antiproliferative effect of SSAs in addition to tumor-related symptom relief has been validated in prospective Phase III trials [14]. The PROMID trial firstly demonstrated this benefit by randomizing 85 patients with grade 1 midgut NETs to octreotide LAR $30 \mathrm{mg}$ versus placebo [35]. However, some questions remained unresolved, such as the role of SSAs in patients with non-midgut and grade 2 tumors, larger hepatic tumor burden and nonprogressive disease. The CLARINET trial was conducted including these subgroups of patients, among others [36]. This Phase III trial included 204 patients with non-functioning NETs (44\% pancreas, $30 \%$ grade $2 \mathrm{Ki} 67 \leq 10 \%$, and $33 \%$ with liver tumor burden $>25 \%$ ) that were randomized to lanreotide autogel $120 \mathrm{mg}$ or placebo monthly. For the whole population, 
the median PFS was not reached for the lanreotide group, and was 18.0 months for the placebo group (HR 0.47; 95\% CI 0.30-0.73, $p<0.001)$. In the subgroup analysis, the median PFS in patients with pNETs was not reached for the experimental arm and was 12.1 months for the placebo arm (HR 0.58; 95\% CI 0.32-1.04, $p=0.0637)$. The results from the open-label extension CLARINET study showed a median PFS of 32.8 months [37].

\section{PROGRESS IN PNET TREATMENT}

Ongoing research around pNETs has showed promising results with novel agents acting through different targets. Although this is a vast improvement, the search for reliable biomarkers and further understanding is still lacking. The anti-apoptotic protein survivin was suggested as a possible predictive biomarker to the dual-targeted therapy, rapamycin and erlotinib [20]. The involvement of the VEGFR2 pathway in NETs was suggested by the PAZONET researchers based on tumor expression and soluble VEGFR2 (sVEGFR2) detection [40, 41]. Decrease in soluble VEGFR2 (sVEGFR2) levels was associated with better PFS compared with patients whose sVEGFR2 levels increased: 12.6 versus 9.1 months, respectively $(p=0.067)$. Other potential predictive biomarkers, such as sVEGFR3, stromal cellderived factor 1 alpha (SDF1- $\alpha$ ), endothelial progenitors or circulating endothelial cells, are still not validated in pNETs [40]. Sensitivity to temozolomide, an inductor of DNA methylation at the $\mathrm{O}^{6}$ position of guanine has been related to low levels of $\mathrm{O}^{6}$-methylguanine DNA methyltransferase (MGMT), an enzyme related to DNA repair [41]. There are two trials that assessed the association of MGMT expression and response to temozolomide- based therapy $(N=21$ and $N=53)$, which showed a median PFS for the group of patients with intact MGMT of 9.25 and 7.5 months, respectively, compared with the group of patients harboring tumors deficient of MGMT (19 and 20 months, respectively) [41, 42]. However, those data are not sufficient to consider MGMT status as an independent predictive factor, as prospective data are required to validate it. At the moment, target expression in NETs is not good enough to predict response; in fact, there is currently no definitive biomarker that could guide our therapeutic decisions in the clinic, not only because of its limited accuracy in sensitivity and specificity, such as with cromogranin A, but also for the technical efforts and costs required for the general applicability of the potential biomarkers proposed at the moment [30].

Some of the following drugs and treatment strategies are currently being investigated, and study results look promising for clinicians to be able to place these agents into treatment algorithms for patients with pNETs.

\section{PEPTIDE RECEPTOR RADIONUCLIDE THERAPY (PRRT)}

The benefit of nuclear medicine in diagnosis and treatment of NETs is also currently being investigated in tumors of pancreatic origin, although the real value of peptide receptor radionuclide therapy (PRRT) in the different NET types and in which line should it be used is unclear [43] (Table 2). PRRT with radiolabeled SSAs ([111In-DTPA0]octreotide, [90Y-DOTA0,Tyr3]octreotide and [177LuDOTA0,Tyr3]octreotate) showed promising outcome results with substantial responses to therapy (ORR $=28-37 \%)$ and prolonged disease control, even following the last dose of 


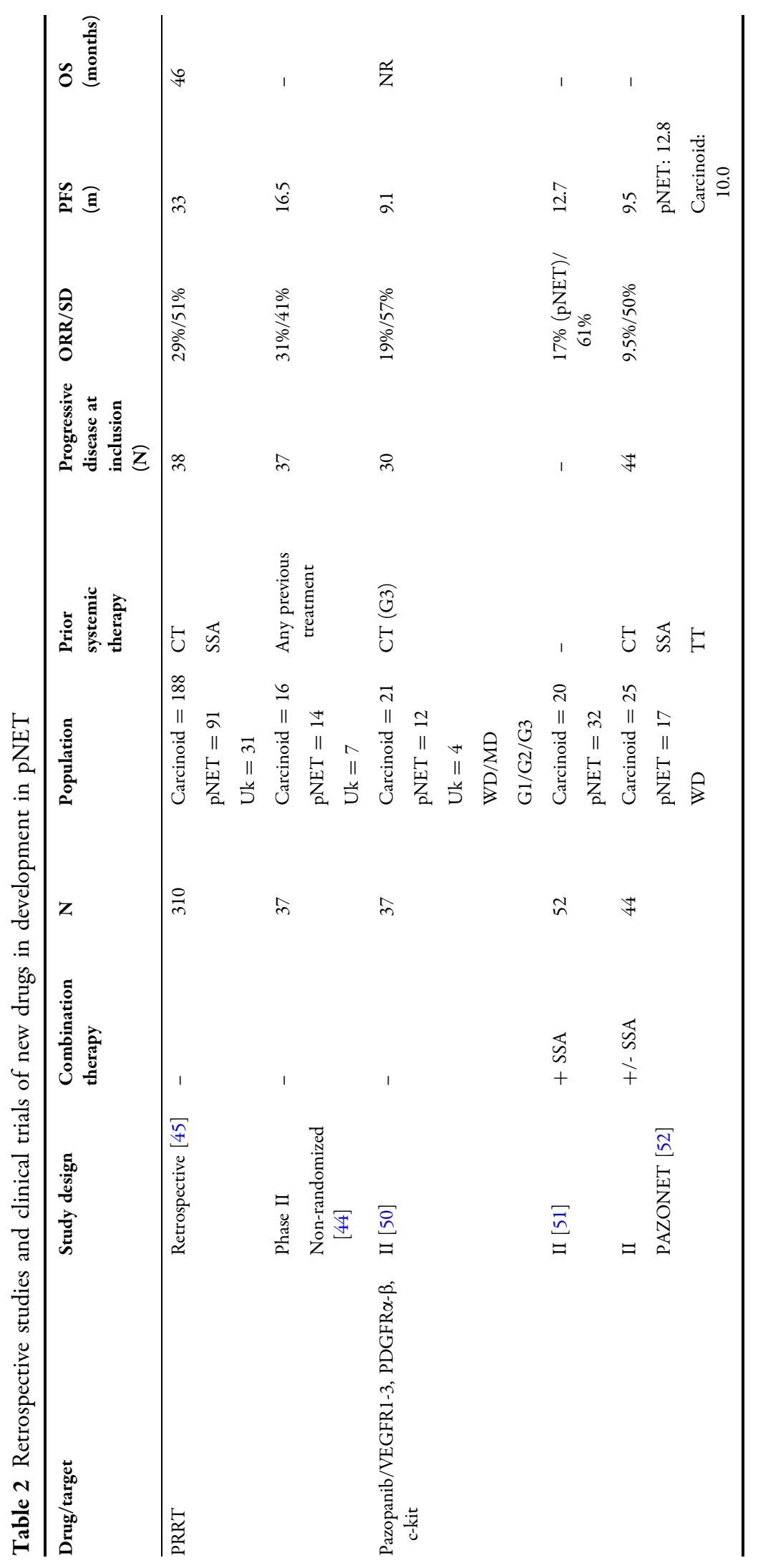




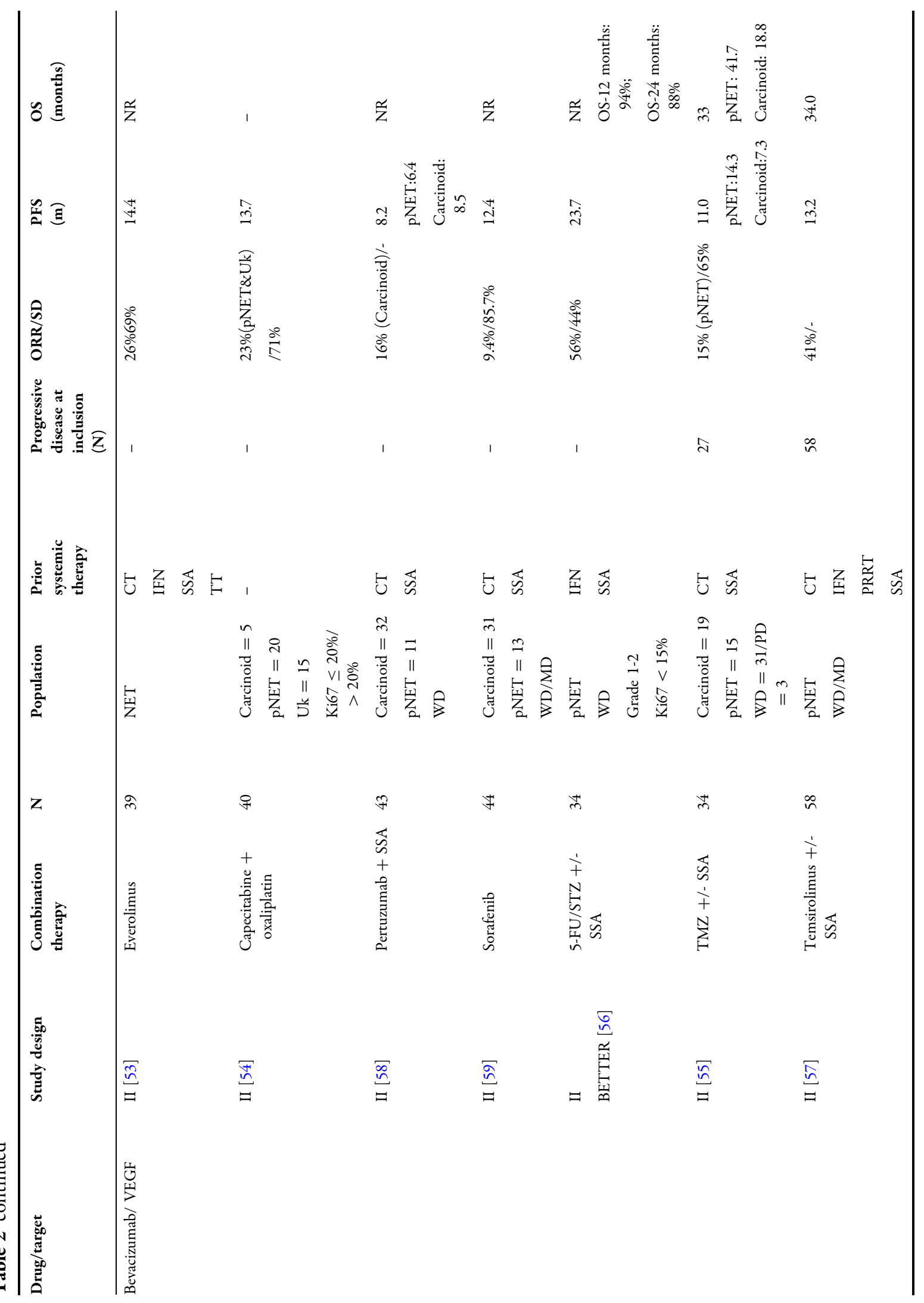




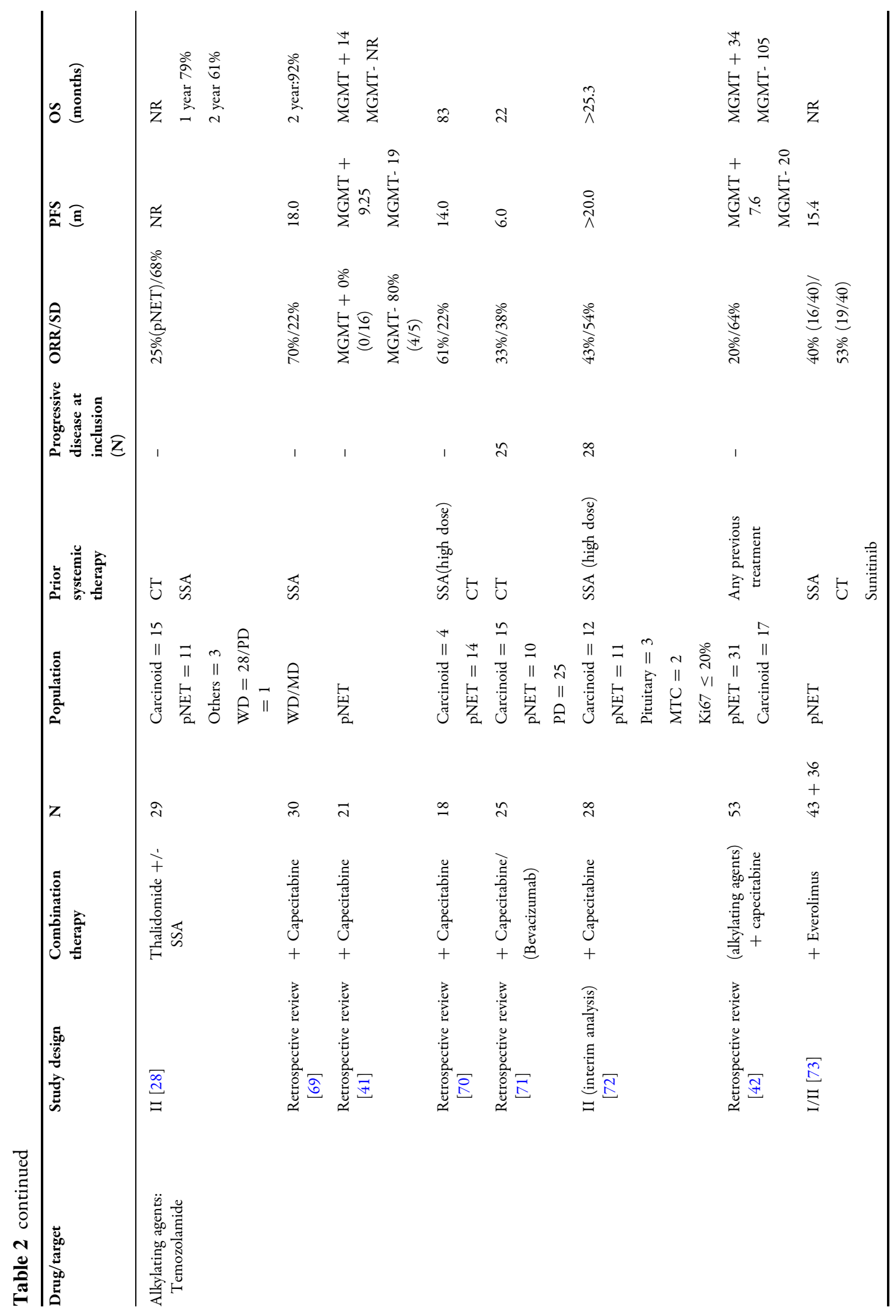




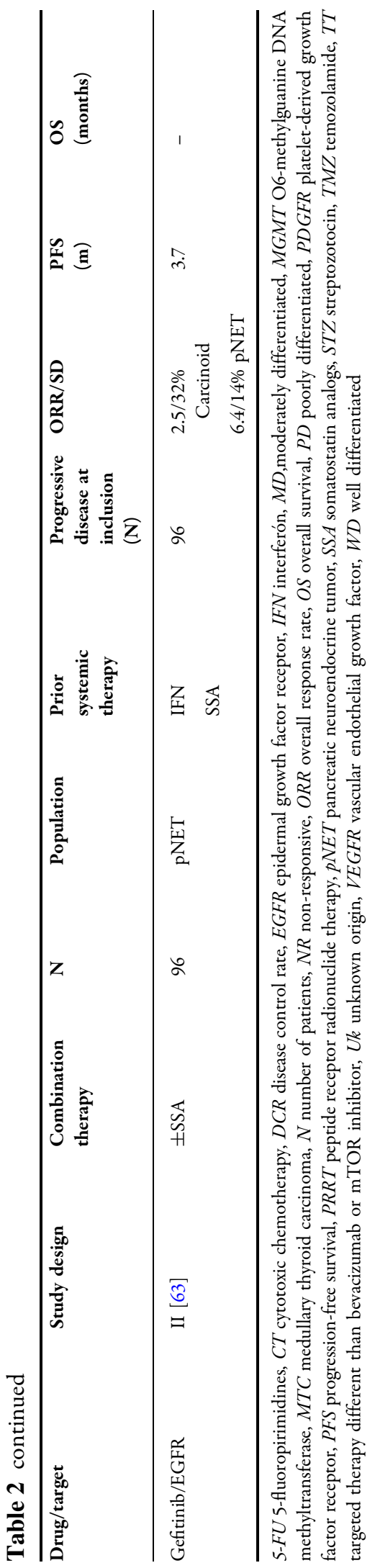

treatment [44]. The least benefit was obtained in patients with poor performance status, significant weight loss, high hepatic tumor burden or bone metastases [45]. According to the low mitotic rate in patients with WD and low-grade pNETs, this treatment strategy can be considered early on in disease progression based on clinical symptoms, serum markers or radiological images to achieve a long-term outcome. However, hematologic adverse events must be taken into account to avoid cumulative toxicity that could limit subsequent treatment lines. In this sense, safety of everolimus after PRRT (median time 18.7 months) was analyzed in a multicenter retrospective study including 24 patients [46]. The more frequent grade $3 / 4$ adverse events were hyperglycemia (12.5\%), thrombocytopenia $(8.3 \%)$ and fatigue (8.3\%). However, data from a compassionate use program showed that patients treated with everolimus after PRRT and chemotherapy had a significant increased risk of toxicity and treatment discontinuation. Grade 3-4 adverse events were described in $87 \%$ of patients [47].

\section{ANTIANGIOGENICS}

As we know, NETs are vascularized tumors with overexpression of VEGF/VEGFR; therefore, new antiangiogenic agents, such as sunitinib among others, are currently being investigated. In a Phase I clinical trial, pazopanib achieved a partial response in a patient with a NET [48]. The mechanism of action of pazopanib and the different selectivity and interaction pattern to other tyrosine kinase inhibitors (TKIs) [49] has been investigated in different clinical settings in Phase II non-randomized clinical trials with and without combination with SSAs, including the PAZONET trial, which was the first study to 
introduce the sequential strategy concept with targeted therapies [50-52].

Bevacizumab, a monoclonal antibody targeting VEGF, has been under research in NETs in combination with other agents with activity in pNETs, such as temozolamide and fluoropirimidines/STZ with or without oxaliplatin and temsirolimus or sorafenib [5559]. The trials achieved an ORR of $9-41 \%$ and median PFS of 11.0-23.7 months. However, there are limitations to these studies, such as small sample size, potential biases in patient selection, different concurrent use of octreotide and the absence of knowledge of the real contribution of bevacizumab in the control of disease. Toxicities in some trials required a dose reduction in $63-80 \%$ of patients [57-59], though the majority of these were easily manageable.

\section{FIBROBLAST GROWTH FACTOR RECEPTOR (FGFR) AND VASCULAR ENDOTHELIAL GROWTH FACTOR RECEPTOR (VEGFR) DUAL INHIBITORS}

The inhibition of the complex fibroblast growth factor (FGF) family has been suggested as a potential strategy to overcome one of the mechanisms of resistance to VEGF/VEGFR inhibitors by FGF activation [60]. Brivanib is a first-in-class dual VEGFR/FGFR TKI that is currently being investigated in hepatocellular and colon cancer. Efficacy of brivanib was investigated in the RIP1-TAG2 mouse model as first- or second-line treatment after single VEGFR2 inhibition (DC101), FGF inhibition (FGF-trap) or VEGFR/PDGFR/RAF inhibition (sorafenib). Brivanib shows activity after failure of first-line VEGFR inhibition, but mainly acts as first-line treatment because of a possible partial blockade of the adaptive mechanisms of evasive resistance [61].

\section{ENDOTHELIAL GROWTH FACTOR RECEPTOR (EGFR) INHIBITORS}

Endothelial growth factor receptor (EGFR) signaling is involved in tumor progression and survival, and has been shown to be a therapeutic target in different tumors [62]. Preclinical data from erlotinib in the Rip1Tag2 mouse model of pNETs showed antitumor activity of growth and angiogenesis [62]. Moreover, concurrent phosphorylation of EGFR and AKT (and S6 ribosomal protein) was described during progression, showing a contribution to PI3 $\mathrm{K}$ pathway promotion due to EGFR activation [20]. Based on the concurrent activation of mitogen-activated protein kinase (MAPK) and PI3 K pathways, preclinical research has been conducted to elucidate the potential benefit of dual therapeutic targeting: e.g., rapamycin and erlotinib. Findings showed promising efficacy results overcoming adaptive resistance of single mTOR inhibitors by the loss of the upstream feedback loop and consequent hyperactivation of AKT [20]. The role of EGFR inhibitors has been investigated in Phase II clinical trials with gefitinib showing activity [63]. Furthermore, there is a currently ongoing Phase II trial investigating patients with low-grade NETs treated with combination therapy of everolimus and erlotinib (clinicaltrials.gov identifier: NCT00843531) (Table 3).

\section{ALKYLATING AGENTS}

Some cytotoxic agents have been investigated in pNETs due to the cumulative evidence showing higher sensitivity of this subtype of 
Table 3 Ongoing clinical trials including pNET

\begin{tabular}{|c|c|c|c|c|}
\hline Study design & $\begin{array}{l}\text { Tumor } \\
\text { origin }\end{array}$ & Treatment & $\begin{array}{l}\text { Primary } \\
\text { endpoint }\end{array}$ & $\begin{array}{l}\text { Trial } \\
\text { registration }^{a}\end{array}$ \\
\hline Phase I & NET & $\begin{array}{l}\text { SNX-5422 mesylate (Hsp90 inhibitor) } \\
\quad+\text { everolimus }\end{array}$ & DLT & NCT02063958 \\
\hline Phase Ib & NET & Sulfatinib & Safety & NCT02267967 \\
\hline Phase I & NET & $\begin{array}{l}\text { Cixutumumab + everolimus } \\
+ \text { octreotide LAR }\end{array}$ & $\begin{array}{l}\text { DLT } \\
\text { PD markers } \\
\text { PK parameters } \\
\text { Safety }\end{array}$ & NCT01204476 \\
\hline $\begin{array}{l}\text { Phase I/II Non- } \\
\text { randomized }\end{array}$ & NET/ACC & TKM-080301 (small interfering RNA) & $\begin{array}{l}\text { DLT } \\
\text { MTD }\end{array}$ & NCT01262235 \\
\hline Phase II & pNET & Dovitinib & 6-month PFS & NCT02108782 \\
\hline Phase I/II & pNET & $\begin{array}{l}\text { Temozolomide }+ \text { pazopanib } \\
\text { hydrochloride }\end{array}$ & $\begin{array}{l}\text { DLT } \\
8 \text { week-RR }\end{array}$ & NCT01465659 \\
\hline $\begin{array}{l}\text { Phase II Non- } \\
\text { randomized }\end{array}$ & NET & Everolimus + erlotinib & ORR & NCT00843531 \\
\hline $\begin{array}{l}\text { Phase II Non- } \\
\text { randomized }\end{array}$ & NET & Gefitinib & 6-month PFS & NCT00075439 \\
\hline $\begin{array}{l}\text { Phase II Non- } \\
\text { randomized }\end{array}$ & pNET & $\begin{array}{l}\text { Capecitabine }+ \text { temozolomide } \\
+ \text { bevacizumab }\end{array}$ & $\begin{array}{l}\text { ORR } \\
\text { Safety }\end{array}$ & NCT01525082 \\
\hline $\begin{array}{l}\text { Phase II Non- } \\
\text { randomized }\end{array}$ & GEP-NET & Lanreotide autogel + temozolamide & 6-month DCR & NCT02231762 \\
\hline $\begin{array}{l}\text { Phase II (NORDIC) } \\
\text { Non-randomized }\end{array}$ & GEP-NEC & $\begin{array}{l}\text { Everolimus }+ \text { temozolomide }(\mathrm{Ki} 67<55 \%) \\
\quad(\text { first line })\end{array}$ & 6-month DCR & NCT02248012 \\
\hline $\begin{array}{l}\text { Phase II (EVINEC) } \\
\text { Non-randomized }\end{array}$ & $\begin{array}{l}\text { GEP- } \\
\text { NET(G3)/ } \\
\text { NEC }\end{array}$ & $\begin{array}{l}\text { Everolimus (after failure of platinum-based } \\
\text { therapies) }\end{array}$ & ORR & NCT00363051 \\
\hline $\begin{array}{l}\text { Phase II (SONNET) } \\
\text { Non-randomized }\end{array}$ & GEP-NET & Lanreotide autogel + temozolomide & 6-month DCR & NCT02231762 \\
\hline $\begin{array}{l}\text { Phase II } \\
\text { (OCLURANDOM) } \\
\text { Randomized }\end{array}$ & pNET & 177Lutetium-octreotate versus sunitinib & 12-month PFS & NCT02230176 \\
\hline $\begin{array}{l}\text { Phase II (CALGB } \\
\text { 80701) Randomized }\end{array}$ & pNET & Everolimus + SSA \pm bevacizumab & PFS & NCT01229943 \\
\hline $\begin{array}{l}\text { Phase II/III } \\
\text { (REMINET) } \\
\text { Randomized }\end{array}$ & $\begin{array}{l}\text { Duodeno- } \\
\text { pNET }\end{array}$ & $\begin{array}{l}\text { Lanreotide autogel versus placebo as } \\
\text { maintenance therapy after response to first } \\
\text { line CT or targeted agents ( } 3-6 \text { months) }\end{array}$ & $\begin{array}{l}\text { 6-month } \\
\text { OS/PFS }\end{array}$ & NCT02288377 \\
\hline
\end{tabular}


Table 3 continued

\begin{tabular}{lllll}
\hline Study design & $\begin{array}{l}\text { Tumor } \\
\text { origin }\end{array}$ & Treatment & $\begin{array}{l}\text { Primary } \\
\text { endpoint }\end{array}$ & $\begin{array}{l}\text { Trial } \\
\text { registration }\end{array}$ \\
\hline $\begin{array}{l}\text { Phase III (SEQTOR) } \\
\text { Randomized }\end{array}$ & pNET & Everolimus $\Rightarrow$ STZ +5 -FU & Second PFS & NCT02246127 \\
Phase III Randomized & pNET & Temozolomide \pm capecitabine & & NCT01824875 \\
\hline
\end{tabular}

$C T$ cytotoxic chemotherapy, $C T C$ enumeration of CTC, $D C R$ disease control rate, $D L T$ dose-limiting toxicities, $G E P$ NET gastroenteropancreatic neuroendocrine tumors, $H s p 90$ heat shock protein 90, LAR long-acting repeatable, $M T D$ maximum tolerated dose, NET neuroendocrine tumor, ORR objective response rate, $O S$ overall survival, $P D$ markers pharmacodynamics markers, $P F S$ progression-free survival, $P K$ parameters pharmacokinetic parameters, $p N E T$ pancreatic neuroendocrine tumor, Second PFS PFS of course $1+$ interval between treatments + PFS of course 2, SSA somatostatin analogs, $S T Z$ streptozotocin

a Clinicaltrials.gov identifer 5-FU fluorouracil

NET to chemotherapy. Temozolomide is an orally available alkylating agent developed as a less toxic drug compared with dacarbazine (DTIC) that showed activity earlier in NETs [24]. The potential role of temozolamide in NETs is based on its capacity for DNA methylation at the $\mathrm{O}^{6}$ position of guanine. Therefore, this agent has been, and is still currently, being studied as a single agent or in combination with a number of different drugs for the treatment of NETs (Tables 2, 3). At the moment, studies investigating temozolomide in combination with thalidomide, based on its antiangiogenic activity against VEGFR and basic FGF (bFGF), found a significant number of infectious complications [64], but in combination with capecitabine (CAPTEM), based on the synergistic activity with temozolomide observed in preclinical research, have demonstrated clinical efficacy and a good safety profile [41]. The cytotoxic effect of alkylating agents of tumor cells with prolonged G0 phase, such as those from WDNETs, is delivered in a time-dependent manner in combination with a continuous exposure to an antimetabolite agent (fluorouracil [5-FU] or capecitabine) or by the MGMT cell depletion conducted by fluoropyrimidines [65] which represent some of the possible synergistic mechanisms.

\section{COMBINATION THERAPIES}

Unfortunately, patients finally progress under initial systemic treatment and subsequent therapies are required as currently there are no agents able to cure the disease. Therefore, current research in NETs also focuses on the mechanisms of intrinsic or acquired resistance that interfere at some point of the disease in the majority of patients. Two major strategies are suggested to overcome resistance in pNETs, such as the combination of targeted therapies with similar mechanisms of action (antiangiogenic approach, such as FGF/VEGF inhibitor [63]), different mechanisms of action (such as temsirolimus or everolimus, and bevacizumab [57]; clinicaltrials.gov identifier: NCT01229943), or by multiple pathway blockade such as (mTOR and EGFR blockade 
[20] or BEZ235 [66]) and by sequencing therapies.

\section{WHAT CAN WE EXPECT IN THE FUTURE FOR THE MANAGEMENT OF PNET?}

We can differentiate five different approaches for the treatment of pNETs, such as local treatments (hepatic-directed therapies, surgery), SSAs, PRRTs, biologically targeted agents and cytotoxic regimens [67]. The selection of the appropriate sequential treatment results in challenging the context of a multidisciplinary approach that becomes essential in this new complex scenario of pNETs.

As it would be hardly possible to conduct a clinical trial for every sequence including all available drugs, surgical and embolization approaches and combination or "watch and wait" strategies, the correct patient stratification based on the improvement of the molecular biology information would help to choose the best treatment option at a precise time of the disease. Understanding molecular behavior based on genetic aberrations resulting in actionable signaling pathways would in decision making.

What have we learnt from the available clinical trials or retrospective series? Considering that most of our treatment decisions in pNETs are based on clinical discernment [67], some guidance can be obtained from clinical studies. Preferential use of SSAs as first-line therapy can be considered in patients harboring WD-pNETs (Ki67 < 10\%) and stable or early and slow progressive tumors. Sunitinib and everolimus have also demonstrated outcome benefit in this subgroup of patients and in those with increased tumor burden, rate of progression and more aggressive histological findings, such as WD-NETs (the role of everolimus in PD-NETs is under research) and in patients showing contraindication to cytotoxic drugs [29, 30]. Whether the maintenance of SSAs beyond progression adds outcome benefit needs to be confirmed in larger clinical trials (this has not yet been done due to its safety profile in combination with other drugs). Results from the PAZONET trial showed benefit of pazopanib in previously treated and targeted-agent naïve patients [68]. In patients with high burden and symptomatic disease, the use of cytotoxic agents for tumor shrinkage may be appropriate as an initial approach. In fact, safety and efficacy between targeted agents (everolimus) and chemotherapy (5-FU + STZ) are being assessed in the SEQTOR trial (clinicaltrials.gov identifier: NCT02246127) (Table 3). At the moment, targeted agents have also shown efficacy in previously treated patients. In the SUN1111 trial, 35-38\% of patients had received previous treatment with SSAs and $66-72 \%$ had received treatment with cytotoxic chemotherapy [29]. In the RADIANT-3 trial, about $50 \%$ of patients had received an SSA and chemotherapy prior to everolimus [33]. However, controversial results have been obtained after treatment with PRRT [46, 47]. Following on from the information obtained from trials investigating SSAs, the PROMID trial only included treatment-naïve patients, and the CLARINET trial included $16 \%$ of patients previously treated $[35,37]$. Finally, PRRT could be considered in patients with WD-NETs with high uptake of tumor lesions on SSTR scintigraphy and not showing potential worse predictive factors, such as clinical deterioration or high tumor burden (e.g., in liver or bone). 
Treatment-related adverse events can also influence our therapeutic decisions, as well as taking into account patients' comorbidities and concomitant treatments. Cytotoxic drug combinations, such as 5-FU, STZ and bevacizumab, showed Grade 3-4 hypertension, abdominal pain and thromboembolic events. Temozolomide-based regimens showed infectious complications due to the immunosuppressive effect due to prolonged exposures for more than 6 months [64] and cumulative toxicities from the temozolomide and thalidomide regimen, whereas $66 \%$ of patients discontinued treatment due to treatment-related toxicity. In the CLARINET trial, $3 \%$ of patients suffered from serious adverse events related to study treatment. The most frequent Grade 3-4 adverse events in the RADIANT-3 trial were stomatitis (7\%), anemia (6\%), hyperglycemia (5\%) and thrombocytopenia (4\%), and in the SUN1111 trial were neutropenia (12\%), hypertension (10\%) and diarrhea and asthenia (5\%) [29, 33, 37].

Treatment goals in pNETs include improvement of survival, relief of tumorrelated symptoms, inhibition of tumor growth, prevention of complications and maintenance of a good quality of life. According to these objectives, particular advantages from targeted therapies in pNETs include prolongation of disease control and survival with an acceptable safety profile that allows these drugs to be administered in a wide spectrum of the disease. Indeed, treatment with these agents is not associated with cumulative toxicity following therapeutic strategies, even in patients remaining on treatment for prolonged periods of time. In this context, combination strategies with 2 or 3 drugs are currently being investigated to try to offer a clinical benefit based on a theoretical synergy between agents that might overcome some mechanisms of escape without a significant increase in adverse events (Table 3). Some examples of these combination strategies include SSA with sunitinib or everolimus, mTOR inhibitors (everolimus/temsirolimus) with bevacizumab, or everolimus with cytotoxic agents (temozolomide). In fact, research around temozolamide-based treatment regimens goes further based on the promising data from retrospective and Phase II clinical trials in WD-NETs and as a salvage therapy in a subgroup of PD-NECs [69-72]. Moreover, based on the benefit observed with targeted agents, with the aim of increasing the tumor response rates, the combination of those drugs with cytotoxic agents, such as temozolomide, is currently being investigated (Tables 2, 3) [73].

In addition to combination strategies, more information around sequencing strategies is required. An ongoing Phase III clinical trial, the SEQTOR trial, would allow the assessment of the real activity of this regimen with updated response criteria and would help assess the right treatment sequence for patients with pNETs, e.g., everolimus followed by streptozotocinfluorouracil or vice versa (Table 3 ).

Finally, new agents in the treatment paradigm of pNETs are also appearing, such as drugs targeting death-domain associated protein/alpha thalassemia/mental retardation syndrome X-linked (DAXX/ATRX). Recent findings in genomic research discovered the role of telomere remodeling genes DAXX/ATRX in NET carcinogenesis with a relatively high mutation rate of $43 \%$ in WD-pNETs [30]. This may be related to a loss of function of p53 in cell cycle progression [74]. Consequently, ongoing early development clinical trials are underway to assess these agents as treatment options in solid tumors. 


\section{CONCLUSIONS}

Treatment sequencing has emerged as a challenging new concept considering the number of effective novel agents that have emerged in the last years for patients with pNETs. Treatment decisions at any time of the disease require enough data to determine the optimal therapeutic strategy to ensure they are not solely based on clinical parameters. Current research is aimed at improving knowledge around the molecular biology of NETs and at assessing different sequencing or combination strategies, including optimum patient stratification in clinical trials that would consequently help when making treatment decisions.

\section{ACKNOWLEDGMENTS}

All authors had full access to all the data in this study and take complete responsibility for the integrity of the data and accuracy of the data analysis. No funding or sponsorship was received for this study or publication of this article. All named authors meet the International Committee of Medical Journal Editors (ICMJE) criteria for authorship for this manuscript, take responsibility for the integrity of the work as a whole, and have given final approval for the version to be published.

Conflict of interest. Enrique Grande has received research grant from GlaxoSmithKline. Teresa Alonso-Gordoa, Juan José Díez, Javier Molina, Pablo Reguera and Olga Martínez-Sáez declare no conflict of interest.

Compliance with ethics guidelines. The analysis in this article is based on previously conducted studies, and does not involve any new studies of human or animal subjects performed by any of the authors.

Open Access. This article is distributed under the terms of the Creative Commons Attribution Noncommercial License which permits any noncommercial use, distribution, and reproduction in any medium, provided the original author(s) and the source are credited.

\section{REFERENCES}

1. Cives M, Strosberg J An update on gastroenteropancreatic neuroendocrine tumors. Oncology. 2014. 28(9):749-56, 758.

2. Strosberg JR, Fisher GA, Benson AB, et al. Appropriateness of systemic treatments in unresectable metastatic well-differentiated pancreatic neuroendocrine tumors. World J Gastroenterol. 2015;21(8):2450-9.

3. Pavel M. Translation of molecular pathways into clinical trials of neuroendocrine tumors. Neuroendocrinology. 2013;97(1):99-112.

4. Singh S, Dey C, Kennecke H, et al. (2014) Consensus recommendations for the diagnosis and management of pancreatic neuroendocrine tumors: guidelines from a Canadian National Expert Group. Ann Surg Oncol (Epub ahead of print).

5. Öberg K, Knigge U, Kwekkeboom D, Perren A, ESMO Guidelines Working Group. Neuroendocrine gastro-entero-pancreatic tumors: ESMO clinical practice guidelines for diagnosis, treatment and follow-up. Ann Oncol. 2012;23(Suppl 7):vii124-30.

6. Ito $\mathrm{T}$, Igarashi $\mathrm{H}$, Nakamura $\mathrm{K}$, et al. Epidemiological trends of pancreatic and gastrointestinal neuroendocrine tumors in Japan: a nationwide survey analysis. J Gastroenterol. 2015;50(1):58-64.

7. García-Carbonero R, Jiménez FP, Teulé A, Barriuso J, Sevilla I. SEOM clinical guidelines for the diagnosis and treatment of gastroenteropancreatic neuroendocrine neoplasms (GEP-NENs). Clin Transl Oncol. 2014;16(12):1025-34.

8. Bosman FT, Carneiro F, Hruban RH, Theise ND. World Health Organization (WHO) classification of tumours of the digestive system, 4th edn. Lyon: IARC, 2010. Available at: http://www.iarc.fr/en/publications/pdfsonline/pat-gen/bb2/. Accessed 23 Jun 2015. 
9. Schillaci O. 18F-DOPA and other radiopharmaceuticals for imaging unknown primary neuroendocrine tumors. J Nucl Med. 2014;55(3):357-9.

10. Has Simsek D, Kuyumcu S, Turkmen C, et al. Can complementary 68 Ga-DOTATATE and 18F-FDG PET/CT establish the missing link between histopathology and therapeutic approach in gastroenteropancreatic neuroendocrine tumors? J N Med. 2014;55(11):1811-1817.

11. Sorbye H, Strosberg J, Baudin E, Klimstra DS, Yao JC. Gastroenteropancreatic high-grade neuroendocrine carcinoma. Cancer. 2014;120(18): 2814-23.

12. Basturk O, Yang Z, Tang LH, et al. The highgrade (WHO G3) pancreatic neuroendocrine tumor category is morphologically and biologically heterogeneous and includes both well differentiated and poorly differentiated neoplasms. Am J Surg Pathol. 2015;39(5):683-90.

13. Susini C, Buscail L. Rationale for the use of somatostatin analogs as antitumor agents. Ann Oncol. 2006;17(12):1733-42.

14. Toumpanakis C, Caplin ME. Update on the role of somatostatin analogs for the treatment of patients with gastroenteropancreatic neuroendocrine tumors. Semin Oncol. 2013;40(1):56-68.

15. Kvols LK, Oberg KE, Dorisio TMO, et al. Pasireotide (SOM230) shows efficacy and tolerability in the treatment of patients with advanced neuroendocrine tumors refractory or resistant to octreotide LAR: results from a phase II study. Endocr Relat Cancer. 2012;19(5):657-66.

16. Kulke MH. Randomized Phase II study to assess the efficacy \& safety of Everolimus + Pasireotide versus Everolimus alone in patients with advanced progressive pancreatic NET. Barcelona, Spain: European Neuroendocrine Tumors Society (ENETS), 2015.

17. Bergers G, Hanahan D. Modes of resistance to antiangiogenic therapy. Nat Rev Cancer. 2008;8(8):592-603.

18. Tijeras-Raballand A, Neuzillet C, Couvelard A, et al. Resistance to targeted therapies in pancreatic neuroendocrine tumors (PNETs): molecular basis, preclinical data, and counteracting strategies. Target Oncol. 2012;7(3):173-81.

19. Ravaud A, Gross-Goupil M. Overcoming resistance to tyrosine kinase inhibitors in renal cell carcinoma. Cancer Treat Rev. 2012;38(8):996-1003.
20. Chiu CW, Nozawa H, Hanahan D. Survival benefit with proapoptotic molecular and pathologic responses from dual targeting of mammalian target of rapamycin and epidermal growth factor receptor in a preclinical model of pancreatic neuroendocrine carcinogenesis. J Clin Oncol. 2010;28(29):4425-33.

21. Izar B, Rotow J, Gainor J, Clark J, Chabner B. Pharmacokinetics, clinical indications, and resistance mechanisms in molecular targeted therapies in cancer. Pharmacol Rev. 2013;65(4):1351-95.

22. Sun W, Lipsitz DS, Catalano P, Maillard JA, Haller D, Eastern Cooperative Oncology Group. Phase II/ III study of doxorubicin with fluorouracil compared with streptozocin with fluorouracil or dacarbazine in the treatment of advanced carcinoid tumors: Eastern Cooperative Oncology Group Study E1281. J Clin Oncol. 2005;23(22):4897-904.

23. Moertel CG, Lefkopoulo M, Lipsitz S, Hahn RG, Klaassen D. Streptozocin-doxorubicin, streptozocin-fluorouracil, or chlorozotocin in the treatment of advanced islet-cell carcinoma. N Engl J Med. 1992;326(8):519-23.

24. Ramanthan RK, Cnaan A, Hahn RG, Carbone PP, Haller DG. Phase II trial of dacarbazine (DTIC) in advanced pancreatic islet cell carcinoma. Study of the Eastern Cooperative Oncology Group-E6282. Ann Oncol. 2001;12(8):1139-43.

25. Kouvaraki MA, Ajani JA, Hoff P, et al. Fluorouracil, doxorubicin, and streptozocin in the treatment of patients with locally advanced and metastatic pancreatic endocrine carcinomas. J Clin Oncol. 2004;22(23):4762-71.

26. Casciano R, Wang $\mathrm{X}$, Stern $\mathrm{L}$, et al. International practice patterns and resource utilization in the treatment of neuroendocrine tumors. Pancreas. 2013;42(2):339-47.

27. Faivre S, Delbaldo C, Vera K, et al. Safety, pharmacokinetic, and antitumor activity of SU11248, a novel oral multitarget tyrosine kinase inhibitor, in patients with cancer. J Clin Oncol. 2006;24(1):25-35.

28. Kulke MH, Lenz HJ, Meropol NJ, et al. Activity of sunitinib in patients with advanced neuroendocrine tumors. J Clin Oncol. 2008;26(20):3403-10.

29. Raymond E, Dahan L, Raoul JL, et al. Sunitinib malate for the treatment of pancreatic neuroendocrine tumors. $\mathrm{N}$ Engl $\mathrm{J}$ Med. 2011;364(6):501-13. 
30. Capdevila J, Meeker A, García-Carbonero R, et al. Molecular biology of neuroendocrine tumors: from pathways to biomarkers and targets. Cancer Metastasis Rev. 2014;33(1):345-51.

31. Yao JC, Phan AT, Chang DZ, et al. Efficacy of RAD001 (everolimus) and octreotide LAR in advanced low- to intermediate-grade neuroendocrine tumors: results of a phase II study. J Clin Oncol. 2008;26(26):4311-8.

32. Yao JC, Lombard-Bohas C, Baudin E, et al. Daily oral everolimus activity in patients with metastatic pancreatic neuroendocrine tumors after failure of cytotoxic chemotherapy: a phase II trial. J Clin Oncol. 2010;28(1):69-76.

33. Yao JC, Shah $\mathrm{MH}$, Ito $\mathrm{T}$, et al. Everolimus for advanced pancreatic neuroendocrine tumors. N Engl J Med. 2011;364(6):514-23.

34. Yao JC, Pavel M, Lombard-Bohas C, Van Cutsem E, Lam D, Kunz T, et al. Everolimus (EVE) for the Treatment of Advanced Pancreatic Neuroendocrine Tumors (pNET): Final Overall Survival (OS) Results of a Randomized, Double-blind, Placebo (PBO)Controlled, Multicenter Phase III Trial (RADIANT3). European Society for Medical Oncology 2014 Congress: Madrid, 2014.

35. Rinke A, Muller HH, Schade-Brittinger C, et al. Placebo-controlled, double-blind, prospective, randomized study on the effect of octreotide LAR in the control of tumor growth in patients with metastatic neuroendocrine midgut tumors: a report from the PROMID Study Group. J Clin Oncol. 2009;27(28):4656-63.

36. Caplin ME, Pavel M, Ćwikła JB, et al. CLARINET Investigators. Lanreotide in metastatic enteropancreatic neuroendocrine tumors. N Engl J Med. 2014;371(3):224-33.

37. Caplin ME, Ruszniewski P, Pavel $M$, et al. Progression-free survival (PFS) with lanreotide autogel/depot (LAN) in enteropancreatic NETS patients: The CLARINET extension study. J Clin Oncol. 2014;32(15_Suppl):Abstr 4107.

38. Grande E, Casanovas O, Earl J, Castellano D, GarcíaCarbonero R, Teulé A, et al. sVEGFR2 and circulating tumor cells to predict for the efficacy of pazopanib in neuroendocrine tumors. J Clin Oncol. 2013;31(15_Suppl):Abstr 4140.

39. Ortega L, Reyes V, Capdevila J, et al. Correlation of VEGFR2 expression in tumor tissue with longer progression-free survival in patients with neuroendocrine tumors (NETs) treated with pazopanib. J Clin Oncol. 2014;32(15_Suppl):e15154.
40. Zurita AJ, Heymach J, Khajavi $M$, et al. Circulating protein and cellular biomarkers of sunitinib in patients with advanced neuroendocrine tumors. J Clin Oncol. 2011; 29(15_Suppl):Abstr 4079.

41. Kulke $\mathrm{MH}$, Hornick JL, Frauenhoffer C, et al. O6methylguanine DNA methyltransferase deficiency and response to temozolomide-based therapy in patients with neuroendocrine tumors. Clin Cancer Res. 2009;15(1):338-45.

42. Walter $\mathrm{T}$, van Brakel $\mathrm{B}$, Vercherat $\mathrm{C}$, et al. O6methylguanine-DNA methyltransferase status in neuroendocrine tumours: prognostic relevance and association with responseto alkylating agents. Br J Cancer. 2015;112(3):523-31.

43. Kwekkeboom DJ, Teunissen JJ, Bakker WH, et al. Radiolabeled somatostatin analog [177Lu-DOTA0, Tyr3]octreotate in patients with endocrine gastroenteropancreatic tumors. J Clin Oncol. 2004;23(12):2754-62.

44. Delpassand ES, Samarghandi A, Zamanian S, et al. peptide receptor radionuclide therapy with 177Lu-DOTATE for patients with somatostatin receptor-expressing neuroendocrine tumors: the first US phase 2 experience. Pancreas. 2014; 43(4):518-25.

45. Kwekkeboom DJ, de Herder WW, Kam BL, et al. Treatment with the radiolabeled somatostatin analog [177Lu-DOTA0, Tyr3]octreotate: toxicity, efficacy, and survival. J Clin Oncol. 2008;26(13):2124-30.

46. Kamp K, Gumz B, Feelders RA, et al. Safety and efficacy of everolimus in gastrointestinal and pancreatic neuroendocrine tumors after (177)Luoctreotate. Endocr Relat Cancer. 2013; 20(6):825-31.

47. Panzuto F, Rinzivillo M, Fazio N, et al. Real-world study of everolimus in advanced progressive neuroendocrine tumors. Oncologist. 2014;19(9):966-74.

48. Hurwitz HI, Dowlati A, Saini S, et al. Phase I trial of pazopanib in patients with advanced cancer. Clin Cancer Res. 2009;15(12):4220-7.

49. Karaman MW, Herrgard S, Treiber DK, et al. A quantitative analysis of kinase inhibitor selectivity. Nat Biotechnol. 2008;26(1):127-32.

50. Ahn HK, Choi JY, Kim KM, et al. Phase II study of pazopanib monotherapyin metastatic gastroenteropancreaticneuroendocrine tumours. Br J Cancer. 2013;109(6):1414-9. 
51. Phan AT, Halperin DM, Chan JA, et al. Pazopanib and depot octreotide in advanced, welldifferentiated neuroendocrine tumors: a multicentre, single-group, phase 2 study. Lancet Oncol. 2015;16(6):695-703.

52. Capdevila J, Castellano D, García-Carbonero R, et al. 'Pazonet': Single-arm multicentric phase II trial of pazopanib in patients with progressive gastroenteropancreatic neuroendocrine tumors (GEPNETs). Eur J Cancer. 2013;49(Suppl 2):Abstr 2589.

53. Yao JC, Phan A, Fogleman D, et al. Randomized runin study of bevacizumab and everolimus in low to intermediate grade neuroendocrine tumors (LGNETs) using perfusion CT as functional biomarker. J Clin Oncol. 2010;28(15_Suppl):Abstr 4002.

54. Kunz PL, Kuo T, Zahn JM, Kaiser HL, Norton JA, Visser BC, et al. A phase II study of capecitabine, oxaliplatin and bevacizumab for metastatic or unresectable neuroendocrine tumors. J Clin Oncol. 2010;28(15_Suppl):Abstr 4101.

55. Chan JA, Stuart K, Earle CC, et al. Prospective Study of bevacizumab plus temozolomide in patients with advanced neuroendocrine tumors. J Clin Oncol. 2012;30(24):2963-8.

56. Ducreux M, Dahan L, Smith D, et al. Bevacizumab combined with 5-FU/streptozocin in patients with progressive metastatic well-differentiated pancreatic endocrine tumours (BETTER trial)-a phase II non-randomised trial. Eur J Cancer. 2014;50(18):3098-106.

57. Hobday TJ, Qin R, Reidy-Lagunes D, et al. Multicenter phase II trial of temsirolimus and bevacizumab in pancreatic neuroendocrine tumors. J Clin Oncol. 2015;33(14):1551-6.

58. Firdaus I, Shih KC, Zakari A, et al. Bevacizumab, pertuzumab and sandostatin for patients with advanced neuroendocrine cancers. J Clin Oncol. 2012;30(15_Suppl): Abstr 4127.

59. Castellano D, Capdevila J, Sastre J, et al. Sorafenib and bevacizumab combination targeted therapy in advanced neuroendocrine tumour: a phase II studyof Spanish Neuroendocrine Tumour Group (GETNE0801). Eur J Cancer. 2013;49(18):3780-7.

60. Chou T, Finn RS. Brivanib: a review of development. Future Oncol. 2012;8(9):1083-90.

61. Allen E, Walters IB, Hanahan D. Brivanib, a dual FGF/VEGF Inhibitor, is active both first and second line against mouse pancreatic neuroendocrine tumors developing adaptive/evasive resistance to VEGF inhibition. Clin Cancer Res. 2011;17(16):5299-310.
62. Nolan-Stevaux O, Truitt MC, Pahler JC, et al. Differential contribution to neuroendocrine tumorigenesis of parallel egfr signaling in cancer cells and pericytes. Genes Cancer. 2010;1(2):125-41.

63. Hobday TJ, Holen K, Donehower R, et al. A phase II trial of gefitinib in patients with progressive metastatic neuroendocrine tumors: A phase II Consortium (P2C) study. J Clin Oncol 2006;24(18_Suppl):Abstr 4043.

64. Kulke MH, Stuart K, Enzinger PC, et al. Phase II Study of temozolomide and thalidomide in patients with metastatic neuroendocrine tumors. J Clin Oncol. 2006;24(3):401-6.

65. Murakami J, Lee YJ, Kokeguchi S, et al. Depletion of O6 methylguanine DNA methyltransferase by O6 benzylguanine enhances 5FU cytotoxicity in colon and oral cancer cell lines. Oncol Rep. 2007;17(6):1461-7.

66. Libutti S. Randomized phase II study to assess safety and objective response rates of BEZ235 versus everolimus in patients with advanced progressive pancreatic NET. Barcelona, Spain: European Neuroendocrine Tumors Society (ENETS), 2015: Abstr L11.

67. Kulke MH. Sequencing and combining systemic therapies for pancreatic neuroendocrine tumors. J Clin Oncol. 2015;33(14):1534-8.

68. Grande PE, Castellano D, García-Carbonero R, et al. PAZONET: Results of a phase II trial of pazopanib as a sequencing treatment in progressive metastatic neuroendocrine tumors (NETs) patients (pts), on behalf of the Spanish task force for NETs (GETNE)NCT01280201. J Clin Oncol 2012;30(15_Suppl): Abstr 4119.

69. Strosberg JR, Fine RL, Choi J, et al. First-line chemotherapy with capecitabine and temozolomide in patients with metastatic pancreatic endocrine carcinomas. Cancer. 2011;117(2):268-75.

70. Fine RL, Gulati AP, Krantz BA, Moss RA, Schreibman S, Tsushima DA, et al. Capecitabine and temozolomide (CAPTEM) for metastatic, welldifferentiated neuroendocrine cancers: The Pancreas Center at Columbia University experience. Cancer Chemother Pharmacol. 2013;71(3):663-70.

71. Welin S, Sorbye H, Sebjornsen S, et al. Clinical effect of temozolomide-based chemotherapy in poorly differentiated endocrine carcinoma after progression on first-line chemotherapy. Cancer. 2011;117(20):4617-22. 
72. Fine RL, Gulati AP, Tsushima DA, Mowatt KB, Bruce JN, Chabot JA. Prospective phase II study of capecitabine and temozolomide (CAPTEM) for pregressive, moderately and well differentiated metastatic neuroendocrine tumors. J Clin Oncol. 2014;32(15_Suppl): Abstr 179.

73. Chan JA, Blaszkowsky L, Stuart K, et al. A prospective, phase $1 / 2$ study of everolimus and temozolomide in patients with advanced pancreatic neuroendocrine tumor. Cancer. 2013;119(17):3212-8.

74. Zhang J, Francois R, Iyer R, Seshadri M, ZajacKaye M, Hochwald SN. Current understanding of the molecular biology of pancreatic neuroendocrine tumors. J Nat Cancer Inst. 2013;105(14):1005-17. 